Contributions intended for this section should be submitted to any of the Co-editors of Acta Crystallographica or Journal of Applied Crystallography. In the letter accompanying the submission authors should state why rapid publication is essential. The paper should not exceed two printed pages (about 2000 words or eight pages of double-spaced typescript including tables and figures) and figures should be clearly lettered. If the paper is available on 5.25" IBM PC compatible or 3.5" Apple/Macintosh diskettes it would be helpful if these could be sent with the manuscript together with details of the word-processing package used. Papers not judged suitable for this section will be considered for publication in the appropriate section of Acta Crystallographica or in Journal of Applied Crystallography.

Acta Cryst. (1990). A46, FC5-FC7

\title{
Comment on 'Magic strains in face-centered and body-centered cubic lattices'
}

\author{
BY BENJAMIN W. VAN DE WAAL \\ Department of Physics CT1324, University of Twente, PO Box 217, 7500 AE Enschede, \\ The Netherlands
}

(Received 2 January 1990; accepted 22 January 1990)

\begin{abstract}
The six symmetry-related so-called magic strain tensors that transform a f.c.c. lattice (or a b.c.c. lattice) into itself, which have been reported recently by Boyer [Acta Cryst. (1989), A45, FC29-FC32] are not unique: an infinite number of displacement tensors can be constructed that transform one lattice into another, or into itself. There is no connection with fivefold symmetry, other than that in any f.c.c. crystal.
\end{abstract}

In a recent paper, Boyer (1989) describes the assignment of special values to the elements of an orthorhombic strain tensor, in order to obtain tensors (termed 'magic') that can transform a f.c.c. or b.c.c. lattice into itself. Since the tensors are symmetric with non-zero off-diagonal elements, it is convenient to use a different frame of reference, $X^{\prime} Y^{\prime} Z^{\prime}$, in which the tensors are diagonal (the axes of Boyer's frame $X Y Z$ are aligned with the cubic axes of the crystal before deformation). As is easily verified, the unit vectors $r^{-1}(1,1,0), r^{-1}(-1,1,0)$ and $(0,0,1)$ (with $r$ $=\sqrt{ } 2$ ) are the eigenvectors of $T$ [defined by Boyer's formula (1)], so $T$ can be diagonalized by the similarity transform

$$
\begin{gathered}
T^{\prime}=R^{-1} T R \\
R=r^{-1}\left[\begin{array}{rrr}
1 & -1 & 0 \\
1 & 1 & 0 \\
0 & 0 & r
\end{array}\right]
\end{gathered}
$$

0108-7673/90/03FC005-03\$3.00

$$
T^{\prime}=\left(a / a_{0}\right)(1+b)^{-1 / 3}\left[\begin{array}{lll}
\lambda_{1} & 0 & 0 \\
0 & \lambda_{2} & 0 \\
0 & 0 & \lambda_{3}
\end{array}\right]
$$

with $\lambda_{1}=1+c, \lambda_{2}=1-c$, and $\lambda_{3}=(1+b) /\left(1-c^{2}\right)$.

Apparently, in the new frame of reference, $X^{\prime} Y^{\prime} Z^{\prime}, T^{\prime}$ represents homogeneous expansions $\lambda_{1}, \lambda_{2}, \lambda_{3}$ in the $X^{\prime}, Y^{\prime}$ and $Z^{\prime}$ directions, respectively, and an overall expansion $\left(a / a_{0}\right)(1+b)^{-1 / 3}$. The orientation of the frame $X^{\prime} Y^{\prime} Z^{\prime}$, relative to $X Y Z$, follows from $R$ : it is obtained by a c.c.w. rotation by $45^{\circ}$ about the positive $Z$ axis, i.e. the $X^{\prime}$ axis is in the original [110] direction. Since, on (110) planes, the atoms are arranged on a rectangular grid with $Y^{\prime}$ spacing $r^{-1} a_{0}$ and $Z^{\prime}$ spacing $a_{0}\left(a_{0}\right.$ is the conventional lattice constant, $c f$. Fig. 1 ), it is clear that a 'magic' strain can be obtained by an interchange of these spacings. Accordingly, the $Y^{\prime}$ spacings should be multiplied by $r$ and the $Z^{\prime}$ spacings by $r^{-1}$. Solving the equations $\left(a / a_{0}\right)(1+b)^{-1 / 3} \Lambda_{i}=1, r, r^{-1}(i=1,2,3)$, for $a, b$ and $c$ leads to the 'magic' values, given by Boyer. Obviously, the strained structure is identical to the original structure after a rotation by $\pm 90^{\circ}$ about the [110] direction. This operation is equivalent to the one given by Boyer - a $45^{\circ} \mathrm{c} . \mathrm{w}$. rotation about $Z$, followed by a $45^{\circ} \mathrm{c}$.w. rotation about the new $X$ - if it is realized that it is not unique: it may be followed (or preceded) by any point-group operation of the cubic lattice. Specifically, if it is followed by a $120^{\circ}$ rotation about the threefold [111] axis (effectively - 1990 International Union of Crystallography 
interchanging the three orthogonal axes), the quoted rotation is seen to be identical to the simple $90^{\circ}$ rotation. The six [110] directions in the cubic lattice allow six equivalent transformations. The exchange of atoms between coordination shells becomes apparent from Fig. 1 . It is clear that, since close contact is transferred from rows to columns, the first neighbours in a row become second neighbours, and the second neighbours in a column become first neighbours, i.e., for example, two $S 1$ go to $S 2$, and vice versa. It is readily verified that the eigenvalues and eigenvectors of the b.c.c. magic strain tensor are identical to those of the f.c.c. tensor, likewise merely describing an interchange of atomic $Y^{\prime}$ and $Z^{\prime}$ spacings in (110) planes. Indeed, a single strain tensor suffices to describe 'magic' strains in f.c.c. and b.c.c. lattices alike.

There are many other ways to transform a f.c.c. lattice into another f.c.c. lattice by a displacement tensor (the term displacement is to be preferred over strain, because the atomic shifts are not small, when compared to the equilibrium interatomic distance). Formally, the two-dimensional grid on any lattice plane can be transformed by homogeneous distortion into the grid on any other lattice plane; proper lateral shifts of successive planes and adjustment of the interplanar spacing will finish the operation. Apparently, this can be done in an infinite number of ways. In general, there will be no simple relation between the orientation of the frame of main axes of the strain tensor, and the crystal axes, however. As an example of other transformations, consider the diagonal tensors with elements $r, 1, r^{-1}$ and $r^{1 / 3}, r^{1 / 3}, r^{-2 / 3}\left(X^{\prime} Y^{\prime} Z^{\prime}\right.$ frame). The first tensor corresponds to a f.c.c. $\rightarrow$ f.c.c. transformation, in which (110) planes become (100) planes. The second corresponds to a f.c.c. $\rightarrow$ b.c.c. transformation (requiring $b=r^{-1}-1$ and $c=0$, i.e. the coordinates of the saddle point in Boyer's Fig. 1); with fixed volume $\left(a=a_{0}\right)$ the b.c.c. structure is in a state of compression that can be relieved by setting $a / a_{0}=2^{1 / 3}(2 / 3) 1 / 2=$ 1.028. Consequently, the barrier height equals the difference in binding energy per atom of a f.c.c. crystal and a b.c.c. crystal, $0.37 \varepsilon$ (Hirschfelder, Curtiss \& Bird, 1967). Diagonal tensors $A$ with elements $1 / 2,1,1\left(X^{\prime \prime} Y^{\prime \prime} Z^{\prime \prime}\right.$ frame with $X^{\prime \prime}$ in the [111] direction) transform f.c.c. into simple cubic as well as simple cubic into b.c.c. Consequently, $A^{2}$ is another f.c.c. $\rightarrow$ b.c.c. tensor.

Since only affine transformations have been considered, there can be no connection with fivefold symmetry, as suggested by the author.
Indeed, the angle $a \cos (1 / 3)=70.53^{\circ}$, which is the angle between close-packed (111) planes in the f.c.c. crystal, is close to $2 \pi / 5$, which allows five equally sized, but differently oriented, tetrahedrally shaped f.c.c. crystals with closepacked faces to be arranged about a common edge, with neighbouring tetrahedra sharing triangular faces (Ogawa \& Ino,1972).

In order to introduce fivefold symmetry in a single f.c.c. crystal, inhomogeneous distortions

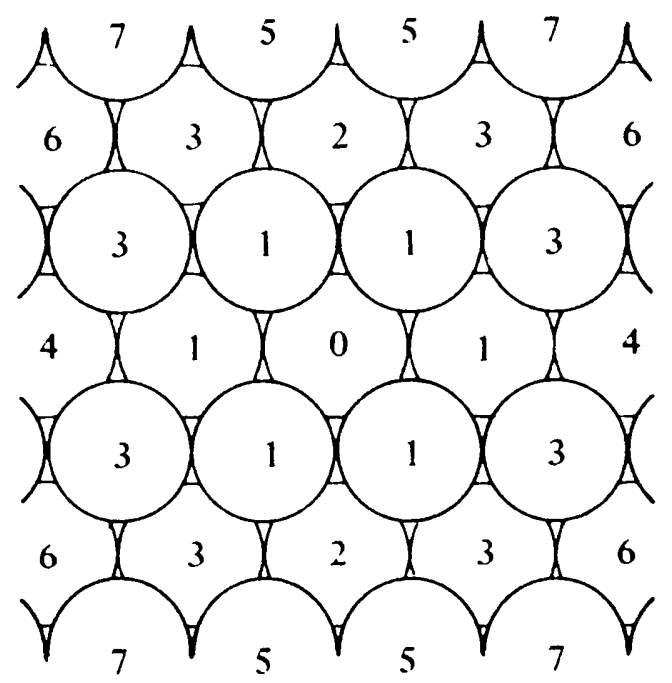

(a)

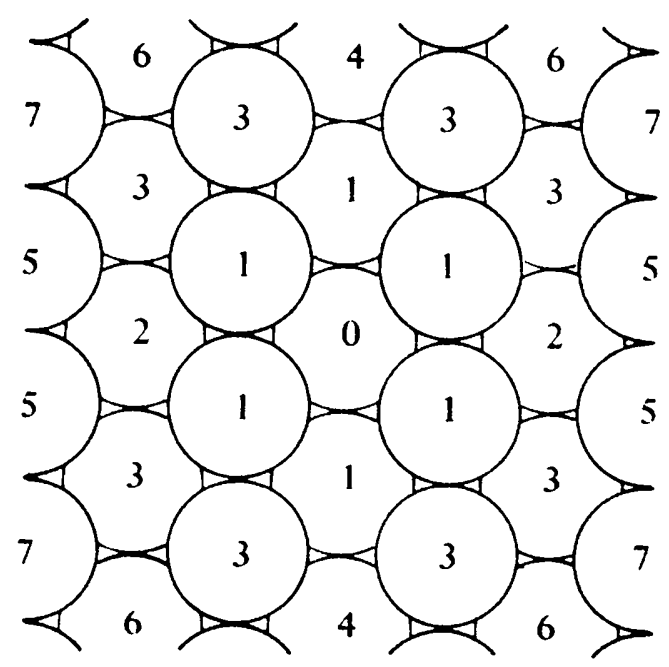

(b)

Fig. 1. (a) Atomic arrangement in two successive (110) layers of a f.c.c. crystal. The view is down the [110] direction $\left(X^{\prime}\right)$. The atoms form close-packed horizontal rows (spacing $r^{-1} a_{0}$ ), with a vertical $\left(Z^{\prime}\right)$ spacing $a_{0} .(b)$ The strained arrangement. The spacings of rows and columns are interchanged. The two arrangements are identical, if the atoms are unlabelled. The numbers identify the coordination shells of a central atom (' 0 '). 
have to be applied that transform a cuboctahedron into an icosahedron (Mackay,1962). The icosahedron, in turn, can be transformed into a f.c.c. cuboctahedron, not necessarily coinciding with the original structure, but related to it by a $2 \pi n / 5$ rotation about one of the fivefold axes of the icosahedron. Since there are two ways to transform the cuboctahedron into the icosahedron, it is clear that the relation with the original orientation of the f.c.c. cuboctahedron is lost after a few transitions.

The physical significance of these transitions (if occurring at all) is restricted to crystals of submicroscopic size (possibly in the nucleation size domain), since the energy barrier connected with the icosahedral transition state becomes prohibitive beyond $N \sim 10^{4}$ atoms (van de Waal, 1989).
The physical mechanism, underlying the possible nucleation of fivefold symmetry in the liquid, has been suggested by Frank (1952), whose ideas have found recent support in molecular-dynamics simulations (e.g. Jonsson \& Andersen, 1988).

\section{References}

Boyer, L. L. (1989). Acta Cryst. A45, FC29-FC32.

Frank, F. C. (1952). Proc. R. Soc. London Ser. A, 215, 43-46.

Hirschfelder, J. O., Curtiss, C. F. \& Bird, R. B. (1967). Molecular Theory of Gases and Liquids, p. 1040. New York: Wiley.

Jonsson, H. \& Andersen, H. C. (1988). Phys. Rev. Lett. 60, 2295-2298.

MaCKay, A. L. (1962). Acta Cryst. 15, 916-918.

OGAWA, S. \& INo, S. (1972). J. Cryst. Growth, 13/14, 48-56.

WAAL, B. W. VAN DE (1989). J. Chem. Phys. 90, 3407-3408. 